\title{
Interpréter l'Europe : éléments pour une relance théorique
}

\section{Christian Lequesne et Andy SMITH}

\section{(2) OpenEdition \\ 1 Journals}

Édition électronique

URL : http://journals.openedition.org/conflits/389

DOI : $10.4000 /$ conflits.389

ISSN : $1777-5345$

Éditeur :

CCLS - Centre d'études sur les conflits lilberté et sécurité, L'Harmattan

Édition imprimée

Date de publication : 15 janvier 1997

ISSN : 1157-996X

Référence électronique

Christian Lequesne et Andy SMITH, «Interpréter l'Europe : éléments pour une relance théorique », Cultures \& Conflits [En ligne], 28 | hiver 1997, mis en ligne le 16 mars 2006, consulté le 30 mars 2021. URL : http://journals.openedition.org/conflits/389 ; DOI : https://doi.org/10.4000/conflits.389

Ce document a été généré automatiquement le 30 mars 2021.

Creative Commons License 


\title{
Interpréter l'Europe : éléments pour une relance théorique
}

\author{
Christian Lequesne et Andy SMITH
}

1 Cette conclusion vise deux objectifs. Le premier est de synthétiser les principaux apports des textes de l'ouvrage, en les resituant notamment par rapport à l'état des travaux sur l'Union européenne que nous avons présentés dans notre article introductif. Plus prospectif, le second objectif consiste à proposer une série de pistes de recherche sur un sujet dont les approches scientifiques méritent d'être en permanence remises sur l'ouvrage. Aussi, le parti pris de ce texte est-il de susciter des interrogations sur les questions conceptuelles que pose l'intégration européenne aux politistes en ne perdant toutefois pas de vue la dimension empirique permettant d'y parvenir. En cela, notre démarche se démarque de celle de certains analystes si enthousiastes à l'égard de l'ingénierie institutionnelle de l'Union européenne qu'ils en oublient d'appuyer leurs écrits sur un terrain ${ }^{1}$. Bien entendu, la modélisation abstraite a un rôle heuristique en science politique mais elle ne peut en aucun cas devenir une raison d'être dans la mesure ou le politique comme la politique ne se résume jamais à des typologies d'acteurs ou de jeux. C'est du reste cette obsession rationalisatrice de la modélisation qui rend à la fois ambitieuse et limitée une bonne partie de la recherche politiste américaine consacrée à l'intégration européenne. Il est donc clair que nous ne prétendons pas tracer des schémas d'analyse totalement novateurs ou encore moins ériger une nouvelle école. Nous souhaitons simplement mettre en avant les éléments d'une démarche pour étudier l'intégration européenne qui, à moyen terme, pourrait s'avérer plus fructueuse que ce qui se pratique souvent actuellement. Le défi conceptuel reconsidéré Nous profitons des contributions de ce numéro pour revenir sur deux questions esquissées dans l'introduction: l'impact de l'intégration européenne sur les rapports entre action publique et système politique, ainsi que sur les rapports entre économie et politique. Sur ces deux questions, les auteurs de ce numéro sont parvenus à des conclusions différentes et quelquefois même contradictoires. Notre propos n'est pas de trancher en faveur de l'une ou de l'autre thèse mais plutôt de signaler les facteurs conceptuels et empiriques qui peuvent être à l'origine des oppositions observées. Politiques publiques communautaires, changement 
et légitimité politique Toutes les contributions à ce numéro s'accordent sur un point qui peut, à première vue, paraître élémentaire mais qui est en fait essentiel: l'intégration européenne ne saurait se résumer aux institutions et aux politiques (au sens de policies) communautaires. Les premières œuvrent dans un environnement politique tellement imbriqué avec les institutions nationales, infranationales et transnationales que leur capacité autonome à " construire l'Europe " est relativement faible. De la même façon, les politiques publiques communautaires sont difficiles à isoler de leurs équivalents étatiques et locaux. Aussi la double question " que change ? " l'action publique communautaire et " comment cela change? " se pose-t-elle avec une acuité particulière. Les auteurs de ce numéro ont répondu à la première interrogation en se démarquant des approches sur les politiques communautaires qui prévalent souvent en Europe et aux Etats-Unis². Il y a en effet un consensus dans ce numéro sur le fait que, du moins pour un politiste, les politiques publiques ne doivent pas être étudiées en tant que telles mais comme des composants de configurations spécifiques de l'action publique ou encore de modèles politiques. De manière plus ou moins explicite, chaque contributeur a ainsi développé une analyse portant sur le rapport entre les évolutions d'une politique publique particulière et ce que Max Weber a nommé les " ordres légitimes de pouvoir "3. Autrement dit, les auteurs ont évité de se noyer dans le détail des dispositifs réglementaires ou redistributifs gérés par l'Union européenne pour étudier les manières dont ceux-ci modifient, déstabilisent voire bouleversent les ordres politiques établis. Ce consensus sur les manières d'aborder le " que change l'action publique communautaire? " s'étiole toutefois, dès lors que l'on aborde la question du " comment ?. En schématisant, deux positions sont en présence. La première est celle du changement par apprentissage. M. Pongy d'une part, S. Rouault et $P$. Muller d'autre part, considèrent que l'intégration européenne implique un apprentissage collectif des acteurs via la constitution de réseaux de médiation. Dans sa contribution sur la crise des services publics en France, Luc Rouban oppose au contraire une analyse selon laquelle les notions d'apprentissage ou de convergence éluderaient la question du changement. Pour L. Rouban, le rapport entre intégration européenne et changement des services publics en France ne peut s'analyser qu'en termes de rupture ou encore de passage d'un modèle institutionnel à un autre par seuils qualitatifs. Il est évident que cette question du changement par apprentissage ou par rupture ne saurait être tranchée une fois pour toutes pour l'ensemble de l'action publique. Chacune des deux formes existe en fonction de l'imbrication plus ou moins grande de la politique en question avec les dispositifs du marché, de la nature des acteurs institutionnels impliqués, et aussi de la manière dont le regard scientifique prend en compte le facteur temps. Références au marché et au secteur privé Nombreuses sont les recherches qui, au début des années quatre vingt dix, ont souligné que l'intégration européenne était dominée par une norme de marché qui irriguait progressivement les systèmes nationaux et locaux de politiques publiques ${ }^{4}$. Plusieurs contributions à ce numéro mettent cependant en cause et/ou reformulent l'idée selon laquelle l'Union européenne serait une simple construction économique sans lien avec le politique. Deux exemples fournissent l'occasion de le discuter directement: ce que L. Rouban appelle la " déréglementation contrôlée " et Laurence Jourdain dans son chapitre, " l'importation " d'une technique de management: l'évaluation. Le premier exemple a le grand mérite de montrer que l'analyse n'est plus au stade d'une opposition tranchée entre le marché unique et une politique industrielle européenne. Au contraire, L. Rouban montre les implications conceptuelles de la fin de l'institution de service public 
en France et de ses équivalents dans d'autre pays. En cela, il problématise la question de la dé- ou de la re-réglementation par rapport aux modèles de politiques publiques. Toutefois, contrôle-t-on vraiment la recomposition de la réglementation au niveau européen? Le schéma d'analyse de G. Majone est en ce sens séduisant ${ }^{5}$, mais qu'est-ce qui le distingue de la gouvernance dite néo-libérale? L. Rouban n'aborde pas cette question de front qui mérite pourtant d'être posée en termes de " gouverne-t-on l'économie européenne ?". La référence à l'évaluation montre que l'influence du privé sur le système politique européen peut être plus subtile. En règle générale, les aides européennes aux régions défavorisées ou à la recherche se font plutôt sur la base de lignes budgétaires qui sont connectées entre elles de manière a posteriori. Dans ce contexte, il n'est guère surprenant que l'évaluation de tels dispositifs administratifs donne rarement lieu à des débats, voire à des décisions politiques. Au final, ces axes d'analyse nous ramènent immanquablement à des questions de fond posées par la science politique. Dans cette perspective, le chapitre de M. Abélès nous fournit un contrepoint stimulant en posant la question : " $\mathrm{y}$-a-t-il à proprement parler un système communautaire? [...] Au chercheur, la construction communautaire pose un intéressant problème, celui d'un modèle d'incertitude qui n'est nullement incompatible avec l'existence d'institutions et de politiques dont la solidité apparaît incontestable ". A partir de ce constat, $\mathrm{M}$. Abélès développe une analyse anthropologique de " la coprésence permanente dans la sphère communautaire de l'actualité immédiate et du projet non encore réalisé ". C'est bel et bien autour de cette idée du temps et de l'inachèvement que nous poursuivons cette conclusion en vue d'esquisser quelques priorités de recherche. Intégration européenne, ordre politique et lien social: un agenda de recherche L'ambition d'un tel exercice est évidemment grande. Elle l'est d'autant plus que, comme cela a déjà été dit, nous croyons que l'étude de l'intégration européenne ne peut pas être séparée d'une réflexion globale sur les sciences sociales. $\mathrm{Si}$ le chemin est long, un certain nombre de chercheurs sont heureusement déjà partis en éclaireurs ${ }^{6}$. Institutions, action publique et pouvoir Les questions que se pose le " nouvel institutionnalisme ${ }^{17}$ ne sont finalement pas très différentes de celles que se posait déjà, au début du siècle, le juriste français M. Hauriou sur les institutions porteuses d'une idée d'oeuvre, mettant au service de cet objectif un pouvoir organisé et devant recueillir le consentement des citoyens ${ }^{8}$. En combinant cette tradition française aux récents travaux de chercheurs britanniques et américains (comme S. Bulmer ${ }^{9}$ ), des questions stimulantes sur les processus d'institutionnalisation au sein de l'Union européenne méritent encore d'être posées. Dans cette perspective, trois champs peuvent être l'objet d'un investissement théorique et empirique. Il conviendrait tout d'abord de réfléchir davantage à la manière dont les institutions communautaires délimitent les modèles institutionnels sectoriels. Il serait ensuite nécessaire de développer des études sur les pratiques politiques au sein des institutions communautaires, car on connaît finalement peu la nature des échanges internes aux commissaires et à leurs cabinets, au secrétariat général du Conseil de l'Union, et plus encore aux membres de la Cour de Justice. Il faudrait enfin s'interroger plus en détails, sur l'influence exercée par des institutions qui restent essentiellement nationales, comme le patronat et les syndicats, sur les configurations de pouvoir en Europe ${ }^{10}$. Polycentrisme, légitimation et représentation politique L'intégration européenne implique également de réfléchir à la conceptualisation du fameux " déficit démocratique " en remettant en question les théories dominantes de la représentation politique. Les trois carences évoquées par B. Jobert à propos de l'Etat-Nation ${ }^{11}$ - 
implication, imputation et interprétation - peuvent constituer un excellent point de départ. L'implication nécessite de revenir absolument sur la question de la représentation des intérêts. Si l'on dispose maintenant d'études très détaillées sur les relations organiques des groupes intérêts avec les institutions nationales et européennes ${ }^{12}$, force est de constater l'absence de véritables travaux sur les effets de ces groupes sur les modèles nationaux et locaux de représentation. Il convient ensuite d'approfondir les problèmes spécifiques posés par un système politique qui est résolument tourné vers la résolution des problèmes " problem solving ". Dans un tel système, les intérêts sociaux sont rarement en phase avec la temporalité et ont dès lors tendance à être déconnectés de la définition de ce qui " pose problème ". L'imputation pose le problème de " qui gouverne? " dans un système politique où la concurrence politique rime souvent avec la dilution de la responsabilité (accountability) et porte atteinte à l'image des représentants politiques comme " gouvernants " ${ }^{13}$. Elle renvoie en outre à une interrogation sur la prégnance du modèle de l'Etat centralisé, l'imputation étant conceptualisée autrement dans les pays plus habitués au polycentrisme politique. L'interprétation exige des travaux que certains qualifieraient de relevant de la culture politique ${ }^{14}$ ou de l'" analyse sociétale "15. Trois axes d'études s'inscrivant dans cette perspective mériteraient d'être développés en particulier. Le premier concerne la dimension symbolique de l'intégration européenne. Si des études récentes nous éclairent sur la difficile émergence des symboles au niveau européen ${ }^{16}$, il demeure la question: comment saisir les effets de cette faiblesse symbolique " dans l'action " ? A notre avis, un moyen consiste à prendre au sérieux l'évocation comme activité politique ${ }^{17}$, $c^{\prime}$ est à dire la manière dont les acteurs se mettent en scène en vue d'exercer un rôle. Les second et troisième axes concernent autant les interprètes que l'activité d'interprétation. Quels sont les rapports entre le système politique communautaire et ces courroies de transmission des débats publics que sont les médias et qui paraissent souvent enfermés dans une représentation " gladiatorienne " de l'Union européenne où s'affrontent les Etats et la Commission? On regrette souvent la faiblesse ou le caractère élitiste des médias européens comme " Arte " ou " Euronews " alors qu'il conviendrait de s'interroger surtout sur le peu de traitement accordé aux enjeux européens par les médias nationaux et locaux. Ce dernier point démontre l'importance d'une réflexion sur la lisibilité des politiques communautaires qui dépasse la simple problématique de la complexité des politiques publiques. C'est autour du triptyque " entendre, comprendre, saisir " que l'on doit essayer de réfléchir au rapport entre l'interprétation de l'intégration européenne et sa légitimation. Les origines de ce que M. Abélès a appelé " l'Europe lointaine "18 sont à rechercher également davantage dans la pratique des institutions communautaires elles-mêmes. A un premier niveau, ce sont les institutions formelles qui se trouvent ébranlées au niveau national par une dynamique qui suscite une série de réactions - adaptation, protestation, reconversion rendant les brouillages plus épais au lieu de les réduire. A un niveau plus profond, c'est la manière dont on conceptualise la politique contemporaine qui est en question. Comment saisir en effet le partage des pouvoirs au sein de l'Union européenne, si l'on s'accroche à une conception de la souveraineté exprimée en termes de hiérarchies indépendantes et verticales? Comment comprendre la diversité des modes de mise en œuvre des politiques communautaires, si l'on s'enferme dans une analyse de la transposition des règlements et des directives communautaires? Des institutions formelles, il est indispensable de passer à l'analyse des institutions sociales qui structurent " les espaces de sens discursifs "19 au sein desquels l'action publique prend 
place. C'est surtout la Commission européenne qui, jusqu'à présent, a fait l'objet d'études dans cette perspective. M. Abélès et I. Bellier nous ont offert une entrée en matière salutaire. Dans un article portant sur " les manières de faire et de penser " au sein de la Commission européenne ${ }^{20}$, ils ont développé la thèse selon laquelle la Commission est un " compromis culturel " qui " engendre dans le champ d'action de cette institution une " culture du compromis " marquée par des orientations et des concepts qui conjuguent plus ou moins heureusement des traditions parfois très différentes "21. De la généralisation de la négociation structurant ce système découlent des " concepts de compromis " qui renforcent la " bizarrerie communautaire "22. Pour approfondir cette analyse, il serait utile d'étudier le refoulement par de nombreux commissaires, parlementaires et ministres européens de leur rôle de représentant de l'Europe. Au final, il ressort sans surprise l'impérative nécessité de préciser le questionnement de l'analyse de la politique contemporaine avec certaines interrogations pérennes des sciences sociales. Espérons que tous ceux qui s'intéressent à l'intégration européenne sont prêts à faire, ou à refaire, ce voyage !

\section{NOTES}

1. Ce travers est par exemple présent dans l'ouvrage de G. Marks et alii, Governance in the European Union, London, Sage, 1996.

2. Voir notre chapitre introductif à ce numéro.

3. Max Weber, Economie et humanisme, Paris, Plon, 1971.

4. B. Jobert (dir.), Le tourant néo-libéral en Europe, Paris, L'Harmattan, 1994.

5. G. Majone, L'Union européenne, un Etat régulateur, Paris, Montchrestien, 1995.

6. K. Middlemass, Orchestrating Europe : the informal politics of European Union 1973-95, London, Fontana Press, 1995.

7. J. March et J. Olsen, Rediscovering institutions : the organizational basis of politics, New York, Free Press, 1989.

8. L. Sfez, Essai sur la contribution du Doyen Hauriou au droit administratif français, Paris, LGDJ, 1966.

9. S. Bulmer, " The governance of the European Union : a new institutionalist approach ", Journal of Public Policy, 13(4), 1993, pp. 351-380.

10. C. Crouch et W. Streeck (dir.), Les capitalismes en Europe, Paris, La Découverte, 1996.

11. Op.cit., 1996.

12. J. Greenwood, Representing interests in the European Union, Basingstoke, MacMillan, 1997.

13. J. Leca, "La gouvernance de la France sous la Ve République : une perspective de sociologie comparative " in F. d'Arcy et L. Rouban (dir.), De la Ve République à l'Europe, hommage à Jean-Louis Quermonne, Paris, Presses de Science Po, 1996.

14. Au sens où B. Badie emploie ce terme dans Culture et politique, Paris, Economica, 1993. 
15. M. Maurice, " Méthode comparative et analyse sociétale. Les implications théoriques des comparaisons internationales ", Sociologie du travail, n² 1989.

16. C. Lager, L'Europe en quête de symboles, Berne, Peter Lang, 1995.

17. M. Abélès, Jours tranquilles en 89. Ethnologie politique d'un département français, Paris, Odile Jacob, 1989 ; et du même auteur, La vie quotidienne au Parlement européen, Paris, Hachette, 1992.

18. M. Abélès, En attente d'Europe, Paris, Hachette, 1996.

19. S. Hall et J. Donal (eds), Politics and ideology, Milton Keynes, Open University Press, 1986.

20. M. Abélès et I. Bellier, " La Commission européenne : du compromis culture à la culture du compromis ", Revue Française de Science Politique, 46(3), juin 1996, p. 431.

21. Op.cit.,p. 432.

22. Op. cit., p. 451.

INDEX

Mots-clés : Relations Internationales, sciences politiques, construction européenne Index géographique : Bruxelles, Europe, Union européenne 